\title{
A Novel Cluster based Routing Algorithm for Hybrid Mobility Model in VANET
}

\author{
D.Rajini Girinath \\ Department of CSE, MNM Jain Engineering College, \\ Chennai, India.
}

\author{
S.Selvan \\ Principal, Francis Xavier Engineering \\ College, \\ Tirunelveli, India.
}

\begin{abstract}
:
This paper proposes a new approach for cluster based routing algorithm for hybrid mobility model to regulating the vehicular traffic. Our approach uses a new hybrid mobility model combining randomwaypoint and group mobility model using static and dynamic sources and a novel cluster based routing algorithm. To transmit the real time updated information and maintain long link duration to improve the data delivery ratio, we propose Location based Multipath Flooding (LMF) algorithm, which is suitable for vehicles with variable mobility. The scenario and the network are modeled and investigated through extensive simulations using Ns2 simulator to study the performance in terms of cluster construction.
\end{abstract}

\section{Keywords:}

VANET, Clustering, Hybrid Mobility, Flooding, Traffic regulation.

\section{INTRODUCTION}

The research on real-time communication in Vehicular Ad Hoc Networks enables distributed applications among vehicular nodes in infrastructure-less areas. Cluster based Routing in VANET is particularly useful for applications that require better routing and scalability to hundreds or thousands of vehicles. Location based clustering techniques can aid in improving the routing performance under different mobility scenarios. Vehicular Ad Hoc Network (VANET) is characterized by vehicles with relatively high mobility and has become a promising field of research paving way to safety of Traffic Management. VANET enables communication between the Vehicles (V2V communications) and the road-side infrastructure (V2I communications) [1].

Static and dynamic cluster heads are responsible for coordination among the nodes within their clusters, and between the clusters. Periodic re-clustering can select nodes with longer travel time and more number of stops like buses act as cluster heads. Network lifetime is prolonged through (i) choosing the optimal path with minimal interference of nodes, (ii) periodic updation in clusterhead regarding the routing and cluster informations, and (iii) routing through the nodes with average speed of nodes. In this work, we present a stand-alone cluster based approach that considers a hybrid and dynamic mobility model. Based on this approach, we present a Location based Multipath Flooding algorithm, which have three primary goals: (i) reducing delay, (ii) prolonging network lifetime by 2-hop cache, and (iii) maximal data delivery ratio at high mobility. Stability in this context implies the need for load balancing and efficient resource utilization. Routing protocols can also employ clustering [2], [3]. In [4], clustering was proposed as a useful tool for locating the destinations. The problem that we address has unique requirements that distinguish it from the energy based load-balancing problem in distributed systems. Real-time communication protocols are inapplicable in an urban mobility model, because we will not derive any standard and repetitive scenarios. In a complete distributed system like VANET, every node can act as a source or a relay node, which motivates the need for efficient algorithms to select servers according to the outlined system goals. Each vehicle should stores the information related to

the cluster within the transmission range of source node. In our model, a fixed number of dynamic and static sources are known to every vehicle of the system, and a static source is always available for processing large amount of data.

In [5], Receiver-oriented approach has been proposed where several nodes may decide to retransmit the message, leading to collisions and/or bandwidth wastage. Each node waits for a random delay before sending the message, except if a neighbor already resent. At high mobility conditions all the nodes move fast and it's very difficult to predict the positions by the source. Because of highly dynamic neighbors this method is not suitable for transmitting emergency/alert messages. In our integrated approach the information pertaining to traffic is maintained in both sources, lesser time in dynamic source and longer duration in static sources for future purpose. If the distance between two cluster head nodes is found to be less than the threshold, the cluster with fewer members is dismissed to reduce communication overheads and its members join other clusters [6]. During high mobility conditions the process of reclustering increases the communication cost. Locating the position of nodes, relative speed predictions and effective communication distance between nodes according to mobility are dealt in our approach. A model is designed through evaluations on large-scale, high-level simulations using 200-nodes and in-depth empirical experiments and the results are analyzed based on the spatial and temporal dependence of nodes. Processes 
like Gauss-Markov and Poisson are being incorporated for routing condition analysis. In addition, we incorporate cluster based routing in various models such as Random Waypoint, RPGM and Manhattan models and compare with our new approach. Our results show that the routing performance may vary drastically across different mobility models and performance of the protocols may vary with different scenarios. This effect can be explained by the interaction of the mobility characteristics with the spatial and temporal dependencies.

Grouping the vehicles can be differentiated in different mobility models according to their spatial and temporal dependencies. For example, two nodes moving in same direction are having high spatial dependency [9]. Temporal dependency is a measure of the relationship between current and previous velocities. High temporal dependency would be spotted between the nodes having the same velocity. VANET's are characterized by high mobility communication in infrastructure-less environments and dynamic topology situations [8], which lead to frequent network partition. VANET's dependency on external parameters like type of the roads, driver's decision, timing, weekdays, and speed of the vehicle and location of the vehicles make it difficult to monitor and manage the entire network. Mobility aware ripple free clusters are used for maintaining stable vehicular infrastructure and inter cluster routing. Thus network can adaptively adjust its dominant routing mechanism based on its mobility features [13].

Our clustering scheme works well in a dynamic environment because it does not require frozen period of motion for initial cluster formation. Hence it is more suitable for urban environments where vehicles change their speed and direction frequently. Vehicular networks are often deployed by the constraint of roadways where trees and buildings influence the practical transmissions as compared to open fields. Vehicle nodes with low relative speed are assigned to the same cluster to tighten the connection in such a cluster. The rest of the paper is organized as follows; Section 2 describes Hierarchical Clustering approach. Section 3 analyzes Hybrid Mobility Model. Section 4 shows the assumptions regarding our novel approach. Section 5 summarizes the simulation setup of our approach. Section 6 describes the performance of our approach and the discussion regarding results.

\section{CLUSTER BASED ROUTING}

Cluster based Routing combines the features of static and dynamic clustering together. Static clusters are formed around the static sources located at the road signals, street corners and congested places known as static clusterhead. However buses are chosen as dynamic sources in our algorithm, having the predefined path and time chart to handle the high mobility situations known as dynamic clusterhead. Hierarchical clustering creates a layering environment that poses some of the main challenges in such ad hoc networks. Top layer consists of static clusterhead, middle layer consists of dynamic clusterhead and lower layer consists of ordinary vehicles. Because of highly dynamic vehicles network topology also changes. This in turn affects the performance of the network and also invokes protocol mechanisms to react to such dynamics. Mobility awareness deals with sudden changes in topology by responding against malfunctions in routing. Some of mobility metrics are considered for cluster construction in order to form a stable cluster structure thereby decreasing its influence on cluster topology. Vehicles Mobility behavior determines the architecture of the cluster. Vehicles are grouped in two different ways either by those vehicles which are in the communication ranges of dynamic sources or by those vehicles which are in the ranges of static sources mounted at traffic signals and road junctions. By doing so, the re-affiliation and re-clustering rate can be naturally decreased.

Dynamic clustering attempts to partition a number of nodes into multi-hop clusters based on the following parameters $(V I D, L I D, s, V L T)$ we defined in Algorithm 1. The (VID,LID, s, VLT) criteria indicate that every vehicle node in a cluster has it own unique $V I D$ and Location $I D$ representing the road in a particular area of the city it belongs. The symbol ' $s$ ' indicates the speed of the vehicle and VLT indicates the vehicles life time in a particular cluster over some period with the probability of $p$, regardless of the hop distance between them. The purpose is to support robust and efficient routing, and adaptively adjust its dominant routing scheme depending on the network mobility manner.

Dynamic clustering scheme, our parameterized clustering scheme requires no periodic re-clustering. As soon as a vehicle enters into the clustering zone its unique VID is registered into the clusterhead and becomes a member of that cluster. Any unclustered vehicle joins a cluster by sending out CJReq message. Mobility also affects the size of the cluster, low mobility increases the size of the cluster compared to high mobility, leading to increase in the number of clusters. A Vehicle can join a cluster if it has a valid $V I D$ and its speed is also an important criterion, if any new vehicle other than ambulance or rescue vehicle enters into the cluster with the speed more than an average speed it is not necessary to update it everywhere. If a vehicle does not receive a response message after a certain period of time, it creates a new cluster and it will become the head for itself, even after that it will send and receive message to become a cluster member or it will continue as a cluster head.

In the present scenario, clustering and routing scheme are run on each vehicle independently without any assistance of other neighboring members. Therefore, even during high mobility, accuracy is maintained. Each vehicle after entering into the cluster region broadcasts control messages [7]. In the algorithm CJM (Cluster Join Messages) for cluster formation is suitable for a network with high mobility, where mobility of vehicles affects the cluster topology. Cluster formation depends only on CJM and not by any other messages thereby overheads are avoided; similar to the idea proposed in [10]. Once the vehicle enters into a cluster region it periodically broadcasts $C C M$ (Cluster Connect Messages) before passing the data, once it receives the response it will start data transmission. In our approach ripple effect of reclustering is reduced by choosing known and defined vehicles like buses as clusterheads and this effect has been reduced in some places by mounting static clusterheads, hence cluster structure and topology has to be maintained smoothly without any force alternations [12]. One advantage of Static clusterhead is gathering accurate neighbor information and cluster structure is promised with specific attributes. Another metric is the duration of each vehicle to become a member of the cluster. By sending a message to all neighbors $(n)$, each vehicle can help each of its neighbors to decide the distance between them. Then each neighbor should send reply information $(r)$, including the $V I D, C I D$, distance, speed and direction. Hence, each vehicle needs to send out $(n+r)$ messages for cluster construction. During cluster construction phase, one 
should consider how to reduce the amount of clustering-related message exchanged for the cluster formation.

From time to time each unclustered vehicle seeks a cluster to join after sending CJM messages periodically, and creates a singlenode cluster to cover itself when there is no proper cluster to join. A too-small cluster, however, may produce large number of clusters and thus increase the length of hierarchical routes, resulting in longer end-to-end delay. To avoid this, two clusters are integrated based on clusterhead speed and by choosing slow speed clusterhead as new head. In our nonoverlapping multihop structure, data transmission is more flexible and do not have a hop limit between two neighboring clusterheads.

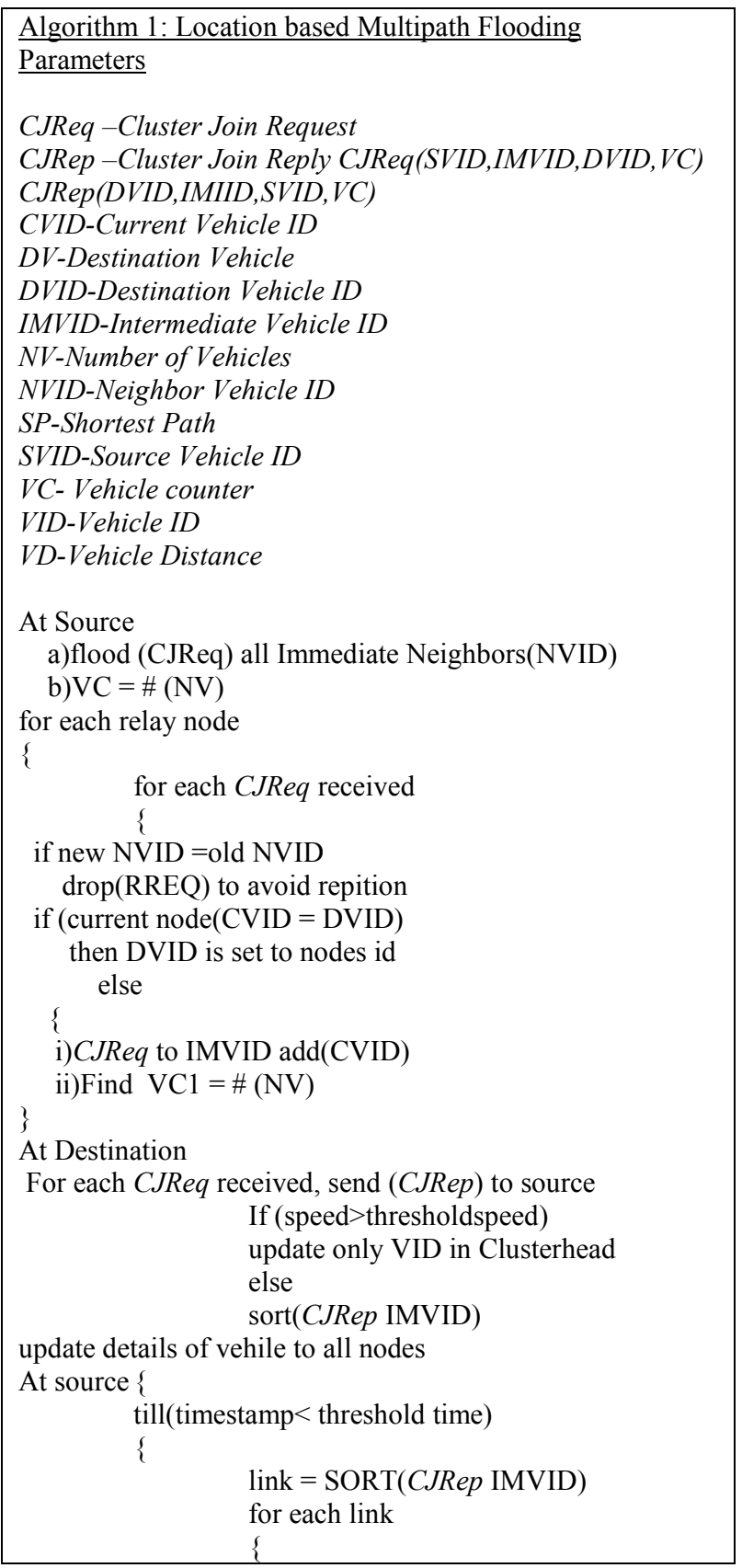

\begin{tabular}{|l|}
\multicolumn{1}{c|}{ find SP= MIN(CJRep VC) } \\
send (data) to DV through SP \\
SORT(CJRep IMVID) \\
for each IMVID in CJRRep \\
$\begin{array}{l}\text { calculate VD } \\
\text { SORT( IMVID) in ascending order of VD } \\
\text { calculate SP \# elements whose VD } \\
\text { store all VID in an array in each cluster }\end{array}$ \\
\end{tabular}

VANET research lies on the development of vehicular communication system that enables convenient, stable and economical distribution of data for the safety and comfort on the road. Dynamic topologies containing highly dynamic nodes will cause the collision on wireless medium. Hence packet delays and losses occur frequently. Each node of VANET acts as a router to transmit the message throughout the network. Consequently, VANET must be a distributed multi-hop network with a timevarying topology [14]. VANET is used in infrastructure-free environments, such as emergency rescue, military and disaster management. Important characteristics of VANET are used in short radio transmission range, low bandwidth, omnidirectional and limited storage capacity. Using clustering techniques we try to minimize topology changes, analyze the data arrival process, minimize the bandwidth utilization, achieve low latency, employ frequent network partition, predict the node location, identify the shortest path and perform link management. On top of the aforementioned salient features and challenges on communication in VANET, a large number of research works have been performed in various phases such as design phase, cluster formation phase, link establishment phase, data transmission phase and traffic control phase. VANET research had focused on the development of various routing protocols, analysis of these approaches under various mobility models, and attempts to manage mobility-related routing issues.

Channel efficiency can be increased by reducing the security overhead. Unsecured network is more dangerous than an overloaded network. Since an accident can be avoided within fractions of a second, reducing the alert message delivery delay is another priority. We try to reduce the redundancy to avoid the delay and utilize the bandwidth efficiently. Our aim is to provide immediate information about a crash to other vehicles. Only the cluster-head vehicles need to send the consolidated safety messages over a channel. It will distribute vague indication of the events that occur during traffic collisions leading to the understanding of the interaction processes. Awareness provides safety driving information and drivers can decide on which alternative path to choose before entering into the critical region of the cluster. It prevents further accidents, traffic congestion and ultimately saves lives and time.

\begin{tabular}{|l|}
\hline Algorithm 2: Cluster Based Routing(CBR) \\
Parameters \\
CID-Cluster ID \\
CH - ClusterHead \\
ns - Source Node \\
nd-Destination Node \\
\hline
\end{tabular}




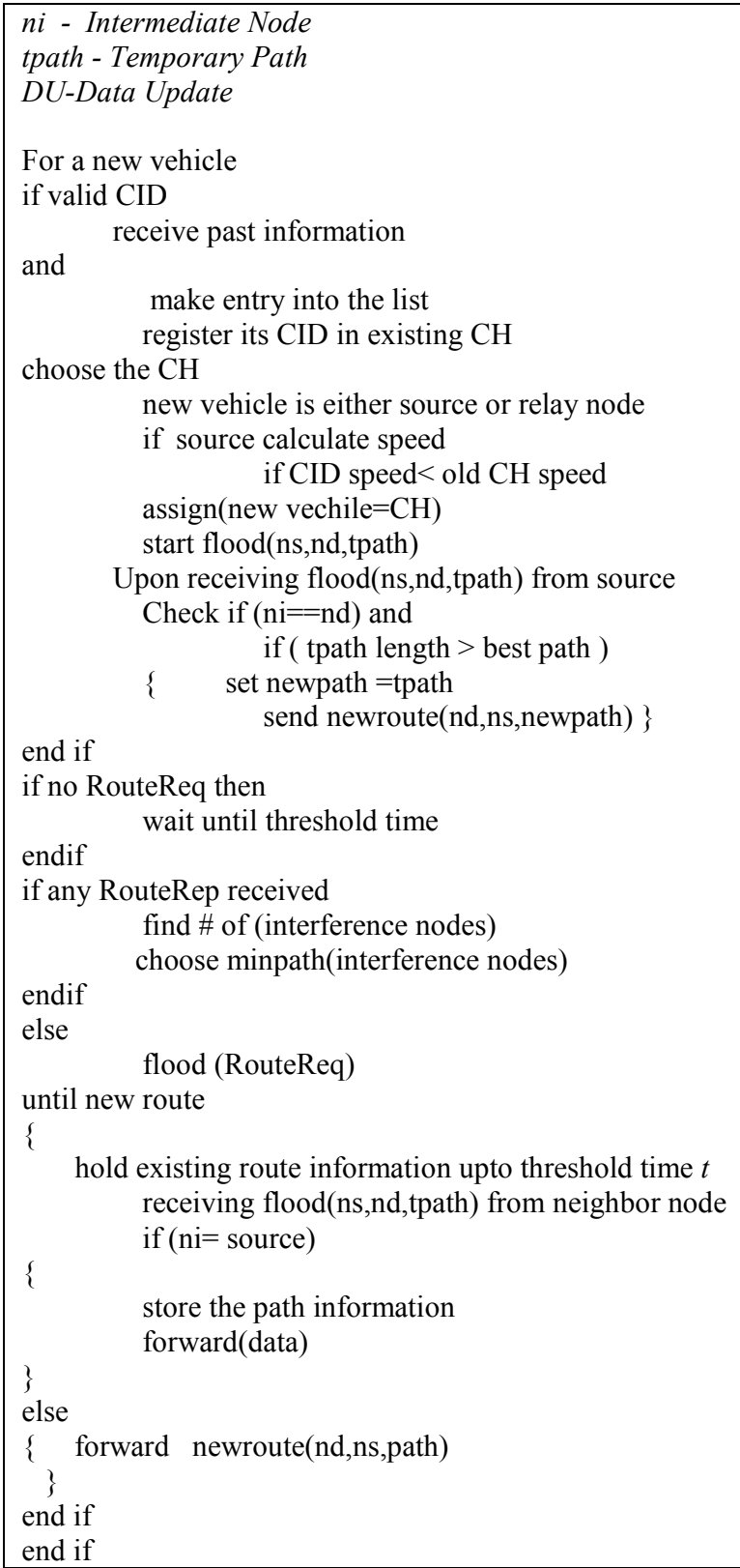
receiving flood(ns,nd,tpath) from neighbor node if $(\mathrm{ni}=$ source $)$

\{ store the path information forward(data)

\} else

\{ forward newroute(nd,ns,path) \} end if

end if

\section{MOBILITY MODEL}

Hybrid mobility model combines several existing mobility models together. These models may also be useful in vehicular adhoc networks. Mobility creates a highly dynamic environment that poses some of the main challenges in such ad hoc networks. The relative movement between nodes creates or breaks connections and changing the network topology. This in turn affects the performance of the network and also invokes protocol mechanisms to react to such dynamics. Hence, mobility modeling becomes crucial to the evaluation and study of ad hoc networking protocols. Traditional mobility models, random walk, random waypoint, manhattan model, gauss markov and reference point group mobility model attempt to mimic the movements of vehicle nodes [17]. In all these randomized models, nodes choose their velocity and direction independently, with no restrictions. The reason for proposing a novel approach for hybrid mobility models is that in many practical scenarios multiple-models may exist within the same network, due to the heterogeneity of nodes and users. The proposed model covers ways in which nodes combine or disperse over time. We also investigate a set of mobility metrics to capture characteristics of mobility. In the hybrid mobility model proposed, the movements of a node will switch from Random Waypoint to Reference Point Group Mobility (RPGM) or vice versa, based on its location in the network or based on the simulation time.

Hierarchical clusters are more stable and have better scalability at low mobility. This type of salutation occurs at non peak duration, only limited vehicles and dynamic sources are moving freely without congestion. So ripple effect of reclustering is somewhat less in low dynamic clustering. Each cluster can accommodate more number of vehicles without affecting the performance and utilizes the resources more efficiently. These cluster based routing protocols attempt to maintain consistent, up-to-date routing information by broadcasting traffic data that requires each node to maintain routing tables to maintain history of the traffic. Network changes are updated throughout the network to maintain the consistent network.

\section{ASSUMPTIONS}

The following assumptions have been made for each cluster in the geographical position involving data transmission and traffic regulation. All the routes in the urban scenario are predefined. The vehicles are chosen as the sources based on the location with the predefined routes and timings. In an urban mobility model buses following the specific route in each region of a city are chosen. Additionally static sources are mounted at the places where traffic congestion are predicted, such as traffic signals, road junctions, hospitals, fire stations, bus stops and tolls. Virtual grid structure is formed across urban model; the static and dynamic sources are mounted and the links between the nodes are easily managed. Because of grid formation high power transmitters are not installed. The vehicle interarrivals follow an exponential distribution. In addition, cluster sizes and interarrival times appear uncorrelated. These Observations agree with traditional modeling of network arrivals as Poisson Process. In our simulation process nodes are added to the cluster according to a Poisson process with rate $\lambda_{1}$. The number of nodes entering into the region is a geometrically distributed random variable with mean value equal to $(N f)$. Similarly data delivery between the vehicles in a cluster and between the two clusters belonging to a particular period is generated according to a Poisson process with rate $\left(\lambda_{2}\right)$. By using the Poisson process we can find out the number of vehicles in a particular area at a time.

During busy hours congestion may occur in certain regions. By using Poisson process maximum number of vehicles that are allowed into the region to avoid congestion are calculated. As per architecture the existing static and dynamic sources calculate the permissible number of vehicles and share the information to maintain the traffic. The probability distribution $N(t)=N_{1}(t)-N_{2}(t)$ gives the distribution of excess vehicles in an interval $t$, where $N_{1}(t)$ is the number of vehicles entering the cluster without congestion in accordance with a Poisson process 
and $N_{2}(t)$ is the number of vehicles leads to congestion inside the cluster. We are using proactive routing approach between the nodes inside the cluster and reactive routing between the clusters. GPS devices are equipped with static and dynamic sources to determine location of the vehicles. DSRC suggesting the transmission range of safety related vehicle-to-vehicle messages is assumed to be 300 meters, and channel contention is resolved using IEEE 802.11 DCF based multi-access control. On road cooperative driving requires a very short-range communication with fast transmission. Routings involved in physical and MAC layer perform different operations. At the time of transmission between vehicles dynamic and unstable Links are established. Nodes may leave and enter into a cluster at any time. The network layer is also involved in VANET for purpose of routing and forwarding. It is observed that at the time of low mobility conditions and less vehicular traffic DSR performs better than AODV, because overhead is somewhat less in DSR. However during high mobility AODV is used to avoid delay [16]. In our work, we focus on performance evaluation, while parameters investigated in the mobility model and mobility characteristics for the following mobility models: Random Waypoint, Manhattan Grid, GaussMarkov, Reference Point Group Mobility (RPGM) model and Static models.

\section{SIMULATION}

Simulations use the ns-2 simulator incorporating both the ns-2 provided implementation of AODV and a modified version incorporating the congestion control algorithm [15]. Nodes follow a random waypoint mobility model, traveling at a variety of speeds. Constant bit rate (CBR) flows are setup between random node pairs. The congestion control parameters in the model are the queue limit and forward limit in a given node. In the original version of DSR there is no forward limit variable and the queue limit is 50 packets. If the queue limits is reached subsequent packets are dropped. The congestion control algorithm requires a route with at least four nodes to become fully active. In a three node route the algorithm may partially operate. To facilitate routes consisting of at least four hops, the transmission range of the nodes and the size of the area in which the simulations are taking place must be considered.

Table 1. Simulation setup

\begin{tabular}{|l|l|}
\hline Parameter & Value \\
\hline Simulation time & 500 seconds \\
\hline Simulation area & $3000 \mathrm{~m}$ X $4000 \mathrm{~m}$ \\
\hline Mobility Models & RWM, RPGM \\
\hline Number of Vehicles & $10-500$ \\
\hline Vehicle velocity & $15-45$ miles per hour \\
\hline Data packet size & 2500 bit \\
\hline Bandwidth & 10 Mbps \\
\hline Beacon packet size & 512 bit \\
\hline Routing Protocol & AODV,DSDV \\
\hline Transmission Range & $250 \mathrm{~m}$ \\
\hline Topologies & $\begin{array}{l}1200 X 1200 \mathrm{~m} \text { Grid with 200mX50m } \\
\text { block size, Real Map }\end{array}$ \\
\hline CBR Sources & 15 sources (4 pkt/sec,64 byte pkt) \\
\hline Performance Metrics & Delivery Ratio, End to End delay, \\
\hline
\end{tabular}

Mobility, Clustering

We also examine the relative routing performance for the two protocols that result from using structured versus un- structured groups. We measure the total number of packet forwards, dropped packets, and received packets. We measure the routing overhead as the number of routing packets sent. Thus, the time interval is set as $250 \mathrm{~ms}$ and considering data items with a fixed size of 2500 bits. Each vehicle sends a beacon message every $0.25 \mathrm{~s}$ to report its own location and speed. Important experiment parameters are listed in Table 1. The performance of the protocols is measured by the following two metrics. Data delivery ratio is the total number of the received nonidentical data items divided by the total number of the disseminated items. Network traffic overhead, the number of bits generated per second, is a summation of individual packet hops. For instance, if a packet of 1000 bits is forwarded ten hops, the network traffic overhead is counted as $20 \mathrm{~K}$ bit-hops. For each measurement, 50 simulation runs are used, and a different seed value is used for each simulation run. For the data delivery ratio, the mean value of the measured data is obtained by collecting a large number of samples such that the confidence interval is reasonably small. In most cases, the $90 \%$ confidence interval for the measured data is less than $10 \%$ of the sample mean.

\section{RESULTS AND DISCUSSION}

The transmitted signal in cluster will affect communications in a neighboring cluster since the radio medium is inherently a broadcast medium. In conditional transmissions relay nodes are selected by the various conditions included in the messages. The receivers are also selected by means of conditions included in the messages, only the nodes that fulfill the conditions will pass the message to their application layers others will discarded the message. Moreover, receiver and transmitter conditions can be different, and in many cases, control messages are required in the neighborhood, so there is a possibility of more number of clusters. In our routing algorithm no such conditions are tested during transmission. We are sending the control messages for communication between the clusters and for checking the validity of the vehicles. By this method the clusters are formed wherever sources are available. Figure 1 and 2 shows the number of clusters hierarchical clustering method and number of clusters in conditional based clustering method respectively.

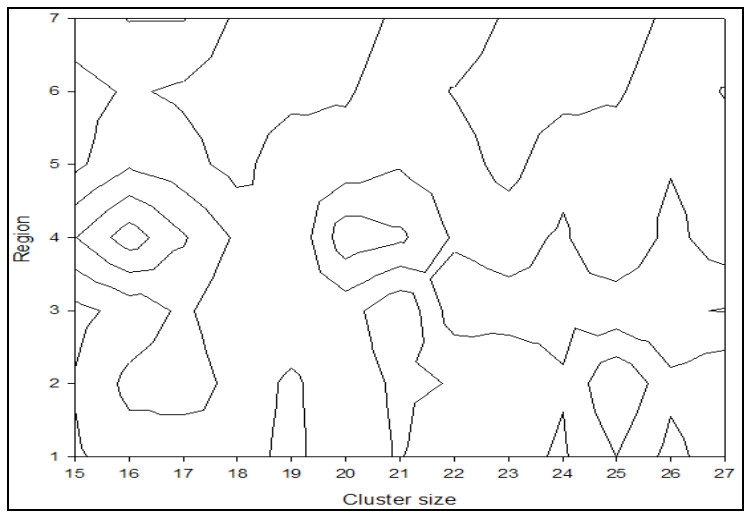

Figure 1. Number of clusters in Hierarchical Clustering method 


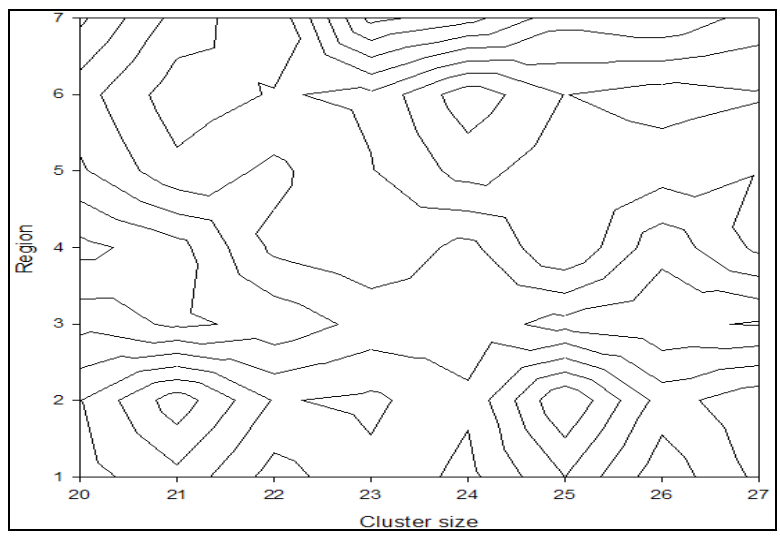

Figure 2. Number of clusters in Conditional based clustering method

For these experiments, we used mean density of ten neighbors per node. The reason is that for lower densities these existing protocols fall into face routing very often. So the overall results for those cases will be mainly determined by the number of times the protocol entered into face routing rather than the neighbor selection itself. Given that the average number of neighbors in our experiments is small. However, we show in our experimental results that the average performance of Hierarchical method is very consistent and scalable across network parameters. We can see that the mean execution time for Hierarchical method is 5 seconds regardless of the density of the network. This is because the proposed routing algorithm is able to compute a good set of neighbors efficiently. Because of dynamic behavior of nodes it will take more time to find the destination. Our grid based clustering method can easily locate the static sources and can easily retrieve the information. Figure 3 and 4 shows restricted distribution of clusterhead in conditional clustering and complete distribution of clusterhead in hierarchical model respectively.

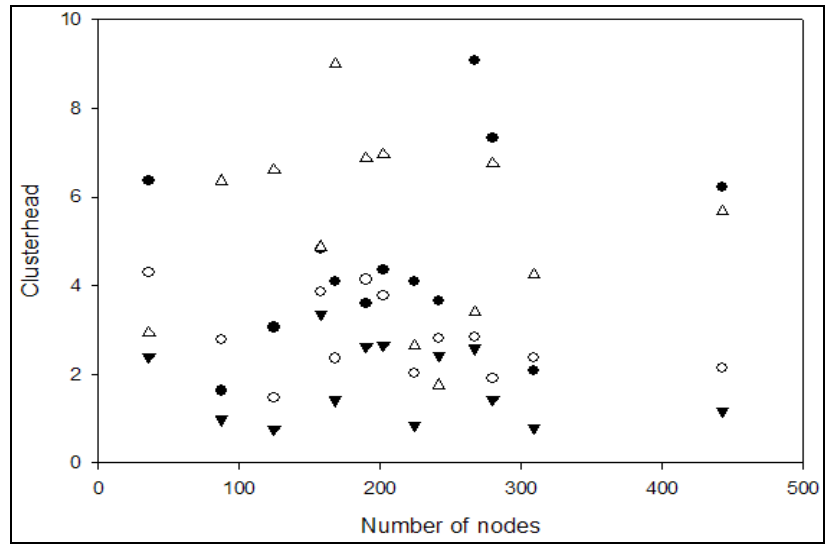

Figure 3. Restricted distribution of Clusterhead in Conditional Clustering

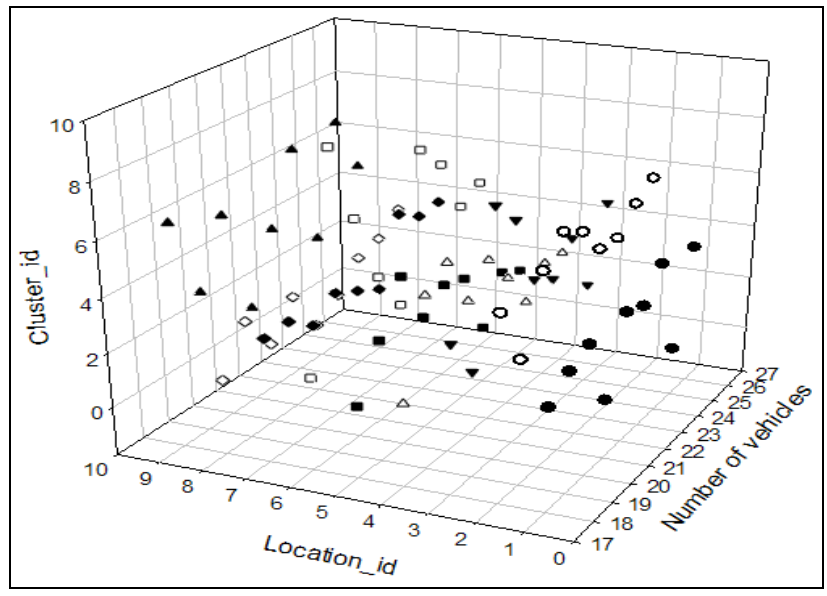

Figure 4. Complete distribution of clusterhead in Hierarchical model

The Random Waypoint Mobility Model is flexible, and it appears to create realistic mobility patterns for the way people might move in, for example, a conference setting or museum. One concern with this model is the straight movement pattern created by the node to the next chosen destination. Because of more randomness we cannot choose this method and it will take more time for computing its location and speed and the number of clusters in a region also increases. The Reference Point Group Mobility Model (RPGM) is a generic method for handling group mobility. An entity mobility model needs to be specified to handle both the movement of a group of nodes and the movement of the individual nodes within the group. Only clusterhead is allowed to interact with other clusters and it will take more time to share the information both within and between the clusters. So further research on mobility models for vehicular ad hoc network protocol evaluation is needed. Our model is developed based on the combination of the best attributes of some of the models. This minimum standard would allow us to evaluate different mobility models more thoroughly. Lastly, we should examine the method used to choose a future location. In other words, the similarities and differences between mobility models that randomly select directions and mobility models that randomly select specific locations should be analyzed. Because of the combination of static and dynamic sources only fixed number of clusters is created as shown in figure 5. Based on our algorithm we can select slow moving buses become a dynamic clusterhead, that can distributed throughout the region. We can predict the arrival time of buses in a region by knowing the predefined time table and speed of the vehicle. Static clusterheads are mounted at the selected locations as shown in figure 6 . 


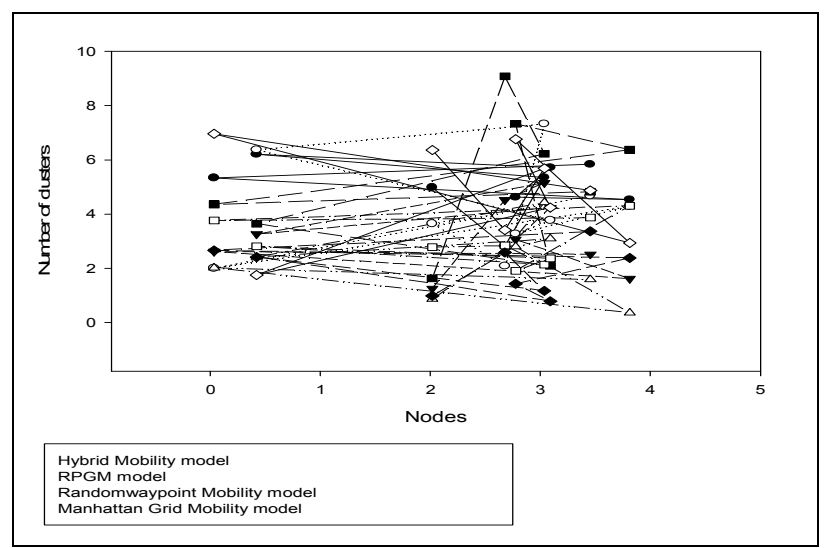

Figure 5. Vehicle movements in different models

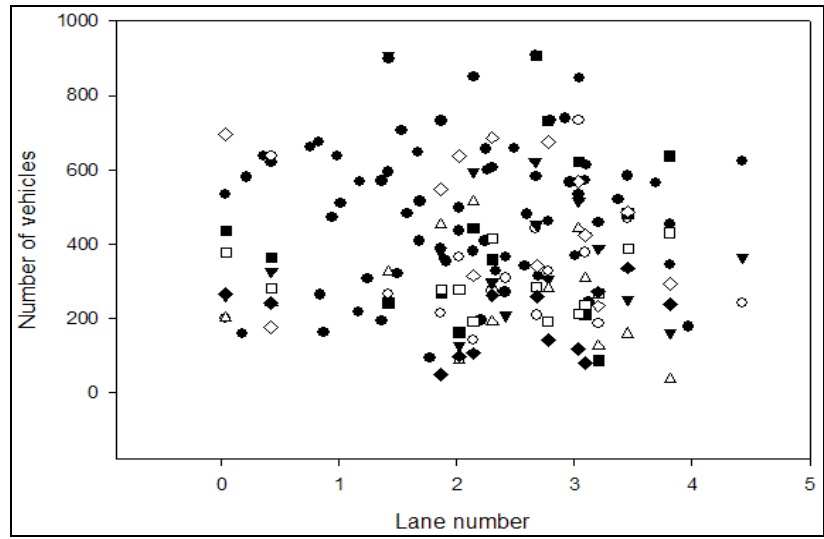

Figure 6. Clusterhead selection process in each region

Figure 7, gives the information regarding the number of stable clusters in each model, In Randomwaypoint model nodes are moving fastly and clusterhead also changing oftenly and hence there are no permanent clusters. In RPGM model even clusters are formed based on conditions, only clusterhead having responsibility to share the information with other clusters. The Gauss-Markov Mobility Model also provides movement patterns that one might expect in the real-world, if appropriate parameters are chosen. In addition, the method is used to force vehicle nodes away from the edges of the simulation area. By using our hierarchical method we form the stable cluster by predefined locations and predefined time table of dynamic sources. Computing the optimal bandwidth consumption is similar to finding the multicast tree with the minimum number of forwarding nodes. In our Hierarchical method routing decision at each single node is performed based on the current network topology. Thus our method is able to adapt to topological changes due to grid formation technique. To support the safety message delivery, we make the necessary modifications in our method by dedicated channel to monitor each vehicle.

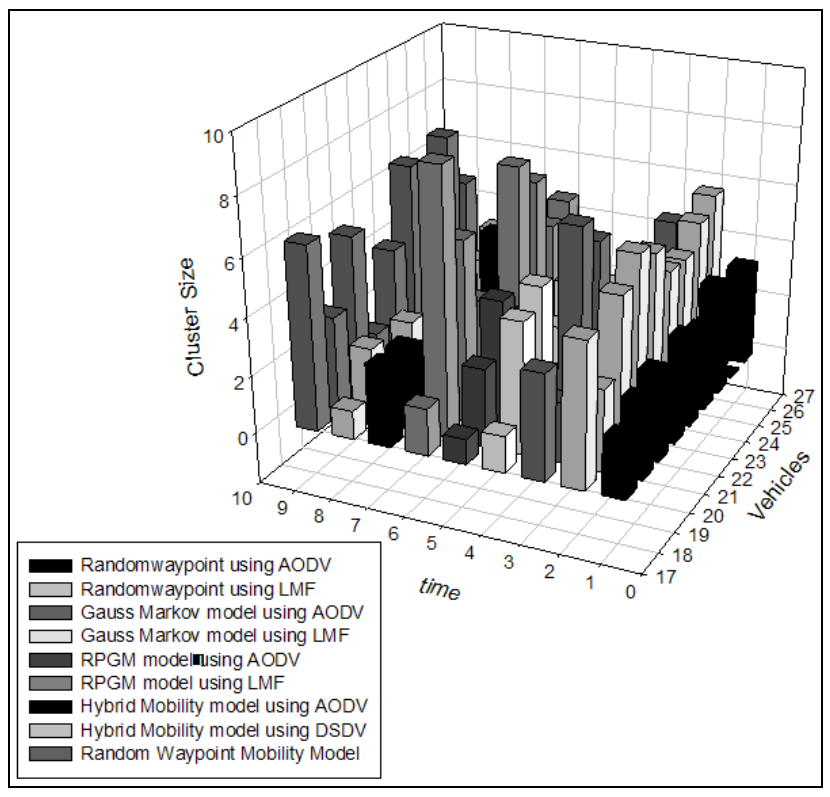

Figure 7. Number of Stable Clusters

Minimizing the link failure avoids reclustering. A nodes response to a link failure event is twofold. First, each node must update its view of the cluster topology and reevaluate the path availability to each of the cluster destinations remaining in the nodes routing table. Second, each node forwards information regarding the link failure to the remaining cluster destinations. When evaluating path availability to destination nodes within the cluster following a topology change, it is necessary to adjust the timing parameter to reflect that the timer has not yet expired. By using the topology information available at each node, the current link availability information is estimated, and maximum availability paths are calculated to each destination node in the cluster. If the node detects that a destination has become unreachable, then the node assumes that the destination has deactivated or otherwise departed from the cluster. In conditional based clustering and data pouring method, there are frequent changes in the links because of checking the conditions frequently leads to link failures. In data pouring method each time it will buffer the data and rebroadcast take more time to reach the destination and need more intermediate nodes to reach the destination.

The random waypoint mobility model contains pause time between changes in direction and/or speed. Once a mobile node (MN) begins to move, it stays in one location for a specified pause time. Figure 6 shows that 2-hop MF has different performance in different mobility models. From Figure 6 we infer that because of random mobility of vehicles, its difficult to deliver the packets continuously. It will take more time to form the stable network and all the nodes need some time for processing the packets. In RPGM mobility model neighbour nodes also assist for the routing process and they travel along with other nodes for some duration but it will need more control messages for cluster formation. By combining the features randomness and grouping behaviour more number of messages is delivered within the stipulated period of time. 


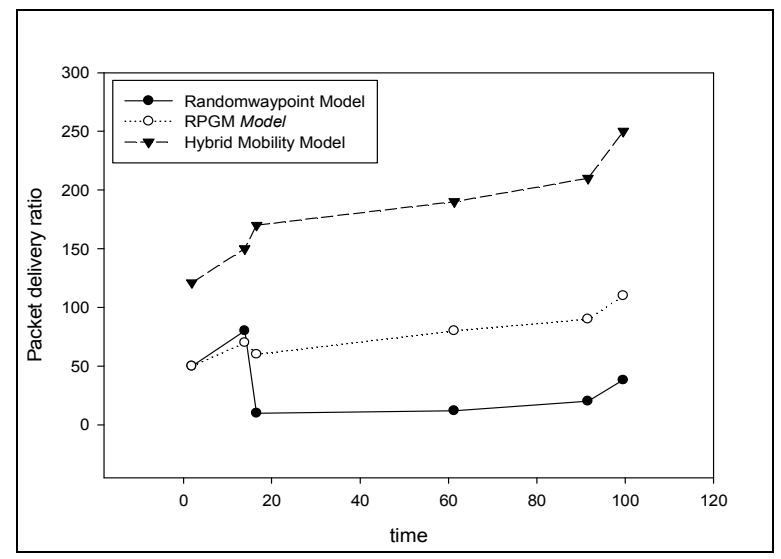

Figure 8. Number of nodes Vs Packet delivery ratio

\section{CONCLUSION}

In this paper, we propose a framework to analyze the impact of cluster based routing on mobility pattern and routing performance of vehicular ad hoc network in a systematic manner. In our, we observe that the combination of clustering and routing pattern does influence the performance of VANET mobility. Our novel design of routing algorithm and Mobility model architecture for traffic regulation reduces the reclustering process and creates more number of stable clusters than other models. The networks are modeled and investigated in various scenarios. Extensive simulation results show that our approach provides better performance in terms of cluster construction, data transmission and vehicular traffic regulation. We believe that several parameters such as traffic patterns, node density and initial placement pattern of nodes may affect the routing performance and need to investigate them further. Our scheme tries to group vehicles nodes with low relative mobility with respect to each other into the same cluster. Thus, the impact of vehicle movement on cluster topology may be minimized.

\section{REFERENCES}

[1] Kun Yang,Shumao Ou, Hsiao-Hwa Chen and Jianghua He," A Multihop Peer-Communication Protocol with Fairness Guarantee for IEEE 802.16- Based Vehicular Networks, IEEE Transactions on Vehicular Technology, Vol. 56, No. 6, pp. 3358-3370,Nov 2007.

[2] Chunhung Richard Lin and Mario Gerla," Adaptive Clustering for Mobile Wireless Networks", IEEE Journal on Selected Areas in Communications, Vol. 15, No. 7, pp.12651275, Sep 1997.

[3] S. Banerjee and S. Khuller, "A Clustering Scheme for Hierarchical Control in Multi-hop Wireless Networks," in Proceedings of IEEE INFOCOM, April 2001.

[4] D. Estrin, R. Govindan, J. Heidemann, and S. Kumar, "Next Century Challenges: Scalable Coordination in Sensor Networks," in Proceedings of the ACM/IEEE International Conference on Mobile Computing and Networking (MOBICOM), August 1999.
[5] Bertrand Ducourthial, Yacine Khaled, and Mohamed Shawky, "Conditional Transmissions: Performance Study of a New Communication Strategy in VANET", IEEE Transactions on Vehicular Technology, Vol. 56, No. 6, pp. 3348-3357,Nov 2007.

[6] Zhigang Wang, Lichuan Liu, MengChu Zhou, and Nirwan Ansari," A Position-Based Clustering Technique for Ad Hoc Intervehicle Communication", IEEE Transactions on Systems, Man and Cybernetics-Part C: Applications and Reviews, Vol. 38, No. 2, pp. 201-208, March 2008.

[7] Jing Zhao, Yang Zhang and Guohong Cao," Data Pouring and Buffering on the Road: New Data Dissemination Paradigm for Vehicular Ad Hoc Networks", IEEE Transaction on Vehicular Technology, Vol. 56, No. 6, pp. 3266-3277,Nov 2007.

[8] Marios D. Dikaiakos, Andreas Florides, Tamer Nadeem, and Liviu Iftode," Location-Aware Services over Vehicular AdHoc Networks using Car-to-Car Communication", IEEE Journal on Selected Areas in Communications, Vol. 25, No. 8, pp.1590-1602, Oct 2007.

[9] Tracy Camp,Jeff Boleng and Vanessa Davies "A Survey of Mobility Models for Ad Hoc Network Research", Vol.2, No.5, pp. 483-502,2002

[10] Tarik Taleb, Ehssan Sakhaee, Abbas Jamalipour, Kazuo Hashimoto, Nei Kato and Yoshiaki Nemoto, "A Stable Routing Protocol to Support ITS Services in VANET Networks", IEEE Transaction on Vehicular Technology, Vol. 56, NO. 6, pp. 3337-3347, Nov 2007.

[11] Jun Peng,Liang Cheng, "A Distributed MAC Scheme for Emergency Message Dissemination in Vehicular Ad Hoc Networks", IEEE Transactions on Vehicular Technology, Vol. 56, No. 6, pp. 3300-3308, Nov 2007.

[12] Kejun Liu,Jing Deng, Pramod K. Varshney and Kashyap Balakrishnan," An Acknowledgement-Based Approach for the Detection of Routing Misbehavior in MANETs", IEEE Transactions on Mobile Computing, Vol. 6, No.5,pp.488-501, May 2007.

[13] Jane Y.Yu and Peter H.J. Chong, "A Survey of Clustering Schemes For Mobile Ad Hoc Networks", IEEE Communications \& Surveys, Vol. 7, No.1,pp.32-48,2005.

[14] Yasser Toor, Paul Muhlethaler, Anis Laouiti and Arnaud De La Fortelle, "Vehicle Ad Hoc Networks: Applications and Related Technical Issues", IEEE Communications \& Tutorials, Vol. 10,No. 3,2008.

[15] Wireless and Mobility Extensions to the ns-2 Network Simulator. CMU Monarch Project. http://monarch.cs.cmu.edu/cmu-ns.html.

[16] K.Daniel Wong, T.J. Kwon, V. Varma, "Towards Commercialization of Ad Hoc Networks ", Proc. of Intl. Conf. on Networking, Sensing \&Control, pp.1207-1211, 2004.

[17] Yenliang Lu, Huier Lin, Yajuan Gu and Ahmed Helmy, "Towards Mobility-Rich Analysis in Ad Hoc Networks: Using Contraction, Expansion and Hybrid Models", IEEE Communications Society, pp. 4346-4351, 2004. 